\title{
Mitochondrial cristae modeled as an out-of-equilibrium membrane driven by a proton field
}

\author{
Nirbhay Patil, ${ }^{1,2}$ Stéphanie Bonneau, ${ }^{2}$ Fréderic Joubert $\odot,{ }^{2}$ Anne-Florence Bitbol, ${ }^{2,3}$ and Hélène Berthoumieux ${ }^{1}$ \\ ${ }^{1}$ Laboratoire de Physique Théorique de la Matière Condensée (LPTMC, UMR 7600), Sorbonne Université, CNRS, F-75005 Paris, France \\ ${ }^{2}$ Laboratoire Jean Perrin (UMR 8237), Institut de Biologie Paris-Seine, Sorbonne Université, CNRS, F-75005 Paris, France \\ ${ }^{3}$ School of Life Sciences, Institute of Bioengineering, École Polytechnique Fédérale de Lausanne (EPFL), CH-1015 Lausanne, Switzerland
}

(Received 6 December 2019; revised 25 June 2020; accepted 27 June 2020; published 5 August 2020)

\begin{abstract}
As the places where most of the fuel of the cell, namely, ATP, is synthesized, mitochondria are crucial organelles in eukaryotic cells. The shape of the invaginations of the mitochondria inner membrane, known as a crista, has been identified as a signature of the energetic state of the organelle. However, the interplay between the rate of ATP synthesis and the crista shape remains unclear. In this work, we investigate the crista membrane deformations using a $\mathrm{pH}$-dependent Helfrich model, maintained out of equilibrium by a diffusive flux of protons. This model gives rise to shape changes of a cylindrical invagination, in particular to the formation of necks between wider zones under variable, and especially oscillating, proton flux.
\end{abstract}

DOI: 10.1103/PhysRevE.102.022401

\section{INTRODUCTION}

Mitochondria are important organelles of eukaryotic cells often called the "powerhouses of the cell," due to their role in the synthesis of adenosine triphosphate (ATP) from adenosine diphosphate (ADP) and an inorganic phosphate $\left(\mathrm{P}_{i}\right)$. These organelles of micrometric size comprise an inner membrane (IM), which delimits a region called the matrix, and an outer membrane $(\mathrm{OM})[1,2]$. The volume between the IM and the OM is called the intermembrane space (IMS). The inner membrane presents numerous tubular invaginations of nanometric size, called cristae, where ATP synthesis takes place. The liquid inside the cristae is isolated from the IMS by the crista junction, an aggregate of proteins that limit the diffusion [3]. Recently, it has been shown that cristae have a higher membrane potential than the intervening boundary membranes, involving confined proton loops and individual functioning of each crista within the same mitochondrion [4]. It has been observed experimentally in isolated mitochondria that the cristae assume different shapes depending on the state of ATP production. Five stationary states (state I to state V) have been introduced to describe the energy status of isolated mitochondria [5], but most present research focuses on state III and state IV, since it allows one to mimic the in vivo situation where an increase of energy demand and energy production occurs. Here we will consider only these two states. A high rate of proton injection by the respiratory chain and of ATP production (state III) is associated with bumpy and wide crista tubules, while a low rate of proton injection and ATP production state IV) is associated with a more regular cylindrical shape as illustrated by Fig. 1. Moreover, recent experiments employing superresolution imaging techniques have directly evidenced the dynamical deformations of cristae in cultivated cells [6].

The endothermic reaction ADP $+\mathrm{P}_{i} \rightarrow$ ATP, is catalyzed by the ATP synthase, which is located in the curved zone of the crista membrane [7]. Traditionally, the ATP synthase enzyme was supposed to use the bulk proton electrochemical potential gradient, involving both the bulk $\mathrm{pH}$ and the electric potential difference between the cristae and the matrix, as energy supply [8]. More recently, it has been established that the proton flux going down the gradient and allowing the rotor to turn with respect to the stator in the ATP synthase enzyme is probably localized on the surface of the crista membrane $[9,10]$. This flux is established and maintained by the respiratory chain, which injects protons from the matrix on the IMS part of the crista membrane. The proteins of the respiratory chain are located in the weakly curved zones of the invagination and thus are spatially separated from the ATP synthases. Recent in vivo $\mathrm{pH}$ measurement show that the $\mathrm{pH}$ decreases along the crista membrane between the proton source (the respiratory chain proteins) and the proton sink (the ATP synthase) [11].

Membrane deformation driven by out-of-equilibrium chemical dynamics is a ubiquitous phenomenon in living cells. A mechanism of hydro-osmotic instabilities generated by ion pumps has been recently suggested to describe the dynamics of the contractile vacuole complex [12]. Surface deformation driven by diffusion of an "active" species is commonly observed in vivo such as the division of eukaryotic cells by accumulations of myosin motors at the cell ring [13]. A phenomenological model has been proposed to study the coupling between diffusion of active agents and surface shape by introducing a modified Helfrich model associated with an active tension coupled to the two-dimensional diffusion of the chemical regulator on the deforming surface [14]. Note that the coupling between a diffusive active agent and the bending of a membrane has not been included in the model [15].

The dynamical coupling between crista shape and ATP production rate is a recent discovery and the physicochemical mechanism at the origin of this coupling still needs to be characterized. The lipid composition of the IM has been pointed out as a key point for the ATP-synthesis machinery. Indeed, the crista membrane is enriched in cardiolipin, and loss of mature cardiolipins affects the shape of the crista and perturbs its function [16]. These lipids possess a protic hydrophilic head, and in vitro experiments have shown that 


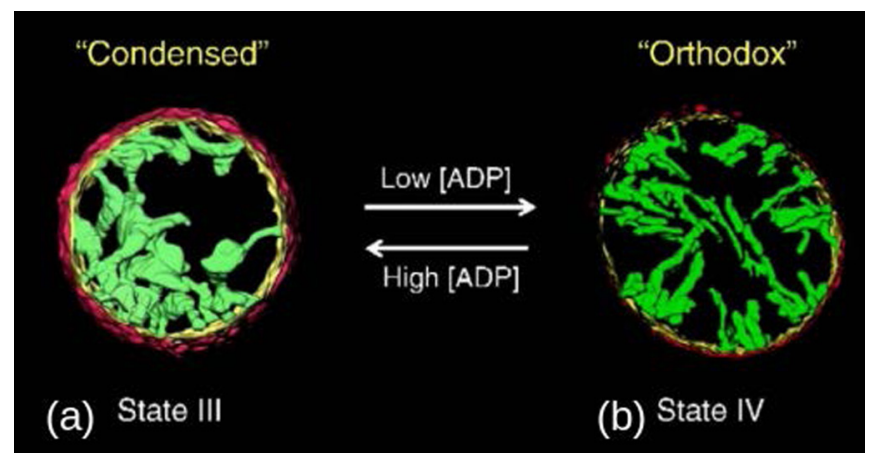

FIG. 1. Cross section of a mitochondrion in different states of ATP production [2]. (a) State III, for which sugar in excess is available leading to a high rate of ATP production. (b) State IV, for which no sugar is available leading to a vanishing ATP production rate. Mitochondria (left to right) have diameters of $1500 \mathrm{~nm}$ and $500 \mathrm{~nm}$.

tubular invaginations can be created by an externally controlled $\mathrm{pH}$ gradient in giant vesicles comprising cardiolipins [17]. A theoretical description modeling these giant vesicles as locally planar bilayer membranes with lipid density and composition heterogeneities in each monolayer [18] has successfully reproduced the dynamics of the membrane in the regime of small deformations [19]. In this model, composition can represent, e.g., the acid and the basic form of cardiolipins, which is controlled by the local $\mathrm{pH}$ field. However, because the crista membrane is enriched in proteins, representing up to $50 \%$ of its mass, a detailed model describing a pure lipid bilayer and including the slippage between the two monolayers may not be necessary to describe this system. Therefore, here we consider a simpler and more phenomenological Helfrich model with $\mathrm{pH}$-dependent parameters.

This work proposes a model for the dynamics of the deformation for the crista membrane between state IV and state III. We start with a reaction-diffusion system describing the proton flux on a cylinder (representing the crista membrane), which contains a proton source, a proton sink, and a reflecting barrier. The resulting proton concentration field will be considered as the driving force inducing the membrane deformation. We then propose a $\mathrm{pH}$-dependent Helfrich model, in which the bending modulus, spontaneous curvature, and tension depend on the local proton concentration, assuming small variations of this concentration. We derive the Green function of the system and study the phase diagram of the crista shape in this model. Finally we solve the hydrodynamic equations of the system for a proton field oscillating between state III and state IV and show that such a model generates dynamical deformations of the membrane, as well as the formation of necks and bumps, along the cylinder leading to a rougher surface in state IV. The last part is devoted to the conclusion.

\section{MODEL OF A MITOCHONDRIAL CRISTA}

\section{A. Proton field along the crista}

We model the crista as an axisymmetric cylinder of membrane of finite length $L$ closed by a spherical cap (see Fig. 2).
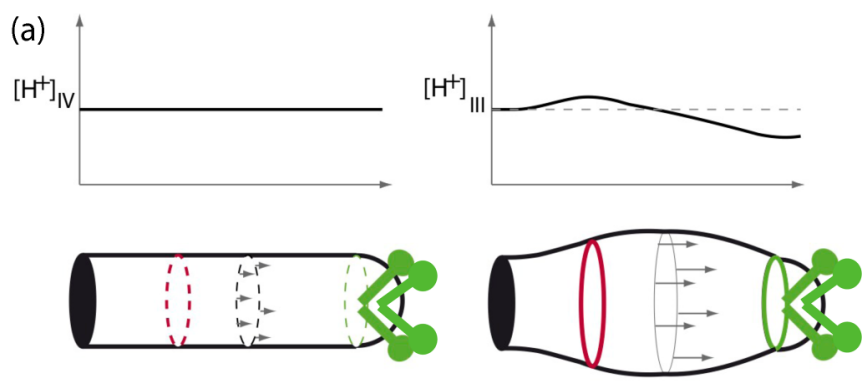

State IV

$$
\begin{aligned}
& \text { - Proton source } \\
& - \text { Proton sink } \\
& \rightarrow \text { Proton flux } \\
& \text { Reflecting boundary } \\
& \text { ATP synthase }
\end{aligned}
$$

State III

(b)

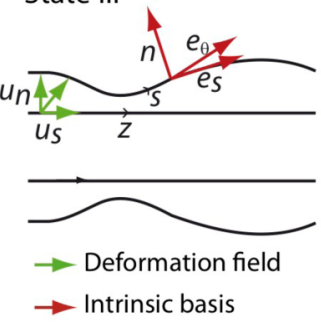

FIG. 2. Schematic representation of a crista. (a) The plots represent the proton concentration on the surface in states III and IV. The tubes represent the shape of the invagination in state III and IV. (b) Deformation fields of the membrane and intrinsic basis of the deformed surface.

In experimental observations [20], cristae feature different shapes, the most common being an elongated pancake, with rows of ATP synthase situated at the rim of the protrusion and respiratory chain proteins located in the flat zone. We nevertheless chose to work in the cylindrical geometry, which is a simple special case of this pancake shape. Indeed, this geometry is analytically tractable, and despite its simplicity, it captures several key ingredients of the system, such as the nanometric confinement and the presence of zones of various curvatures. The protons diffuse on the crista surface at the concentration

$$
\left[\mathrm{H}^{+}\right](s)=\left[\mathrm{H}^{+}\right]_{\mathrm{IV}}+h(s)
$$

where $s$ is a coordinate parametrizing the position along the tube, while $\left[\mathrm{H}^{+}\right]_{\mathrm{IV}}$ represents the constant concentration in state IV taken as a reference, and $h(s)$ is the variation in the proton concentration induced by the functioning of the respiratory chain and the ATP synthases. Note that the concentration is expressed as a number of protons per unit of length, taking advantage of the one-dimensional symmetry of the problem. At one end of the cylinder, $s=0$, one finds a reflecting barrier for the protons modeling the crista junction, while at the other end, $s=L$, a ring-shaped proton sink models the ATP synthases. Between the two, at $s=L_{s}$, a ring-shaped proton source models the respiratory chain. This model for the geometrical confinement of the ATP synthesis machinery takes into account the spatial separation of the proton source and sink and their respective localization in zones of low and high curvature [20].

We assume that $h(s)$ is small, i.e., $h(s) /\left[\mathrm{H}^{+}\right]_{\mathrm{IV}} \ll 1$, and that the tube shape does not deviate much from a regular cylinder. In this framework, the equation governing the proton 
TABLE I. Parameter values estimated from recent experimental measurements.

\begin{tabular}{lccc}
\hline \hline$L$ & $150 \mathrm{~nm}$ & $R$ & $10 \mathrm{~nm}$ \\
$k_{s c}$ & $600 \mathrm{~s}^{-1}[11]$ & $k_{s k}$ & $2.7 \times 10^{4} \mathrm{~s}^{-1}[11]$ \\
$D$ & $10^{-7} \mathrm{~cm}^{2} \mathrm{~s}^{-1}[9]$ & $\omega$ & $0.63 \mathrm{~s}^{-1}$ \\
$\sigma_{0}$ & $10^{-7} \mathrm{~N} \mathrm{~m}^{-1}[19]$ & $\kappa_{0}$ & $10^{-19} \mathrm{~N} \mathrm{~m}^{-19} \mathrm{~N} \mathrm{~m} \mathrm{~m}^{-1}[19]$ \\
$\eta_{b}$ & $10^{-9} \mathrm{~N} \mathrm{~s} \mathrm{~m}$ & $5 \times 10^{-10} \mathrm{~N} \mathrm{~s}^{-19}$ \\
\hline \hline
\end{tabular}

concentration on the surface can be written as follows:

$$
\begin{gathered}
\frac{\partial h(s, t)}{\partial t}=D \frac{\partial^{2} h(s, t)}{\partial s^{2}}+S_{\text {in }}(s, t)-S_{\text {out }}(s, t) \\
\left.\frac{\partial h(s, t)}{\partial s}\right|_{s=0}=0
\end{gathered}
$$

where Eq. (3) illustrates the reflecting barrier, $D$ is the diffusion coefficient, and $S_{\text {in }}(s, t)$ and $S_{\text {out }}(s, t)$ are the source and the sink of the proton, respectively. We consider a system oscillating with a period $T=2 \pi / \omega$ between a state IV of homogeneous proton concentration and a state III associated with a maximal proton flux and write

$$
\begin{aligned}
S_{\text {in }}(s, t)= & \frac{k_{s c}[1-\cos (\omega t)]}{2 \sqrt{2 \pi \Delta_{1}^{2}}} \exp \left[\frac{-\left(s-L_{s}\right)^{2}}{2 \Delta_{1}^{2}}\right], \\
S_{\text {out }}(s, t)= & \frac{k_{s k}[1-\cos (\omega t)]}{2 \sqrt{2 \pi \Delta_{2}^{2}}}\left[\left[\mathrm{H}^{+}\right]_{\mathrm{IV}}+h(s, t)\right] \\
& \times \exp \left[\frac{-(s-L)^{2}}{2 \Delta_{2}^{2}}\right],
\end{aligned}
$$

with $k_{s c}$ the maximal rate of injection of the proton source, $k_{s k}\left(\left[\mathrm{H}^{+}\right]_{\mathrm{IV}}+h(s, t)\right)$ the maximal rate of the proton sink. The spatial extensions of the source and of the sink are modeled by two Gaussian functions of widths $\Delta_{1}$ and $\Delta_{2}$, respectively. The system oscillates between state IV $\left(S_{\text {in }}(s, t)=0\right.$, $\left.S_{\text {out }}(s, t)=0\right)$ for $t_{\mathrm{IV}}=0$ modulo $\mathrm{T}$, noted [T], and state III (where the source and the sink function at their top rates) for $t_{\mathrm{III}}=T / 2[T]$ with a frequency $\omega$. We assume that there is no proton accumulation in the cristae during a period, i.e., $\int_{0}^{L} d s \int_{0}^{2 \pi / \omega} d t S_{\text {in }}(s, t)=\int_{0}^{L} d s \int_{0}^{2 \pi / \omega} d t S_{\text {out }}(s, t)$, which sets the value of the ratio $k_{s c} / k_{s k}$.

The proton concentration $h(s, t)$ is obtained by solving numerically Eq. (3) with an initial vanishing concentration field $h(s, 0)=0$, using the parameter values given in Table I. The profiles of the proton concentration along the tube at different times, shown in Fig. 3, are obtained assuming a diffusion coefficient of $D=10^{-7} \mathrm{~cm}^{2} \mathrm{~s}^{-1}$ [9], which corresponds to the estimated diffusion coefficient of protons along a lipid membrane. The system oscillates between state IV, in which the source $S_{\text {in }}(s, t)$ and the sink $S_{\text {out }}(s, t)$ vanish and the field $h\left(s, t_{\mathrm{IV}}\right)$ is uniform and equal to zero along the cylinder, and state III, for which the proton concentration is approximately homogeneous between the junction $(L=0)$ and the source $\left(L=L_{s}\right)$ and decreases between the source and the sink. Note that protons diffuse with a characteristic time $\tau_{D}=L^{2} / D=$ $0.25 \mathrm{~ms}$ between the source and the sink. An injection rate $k_{s c}$ equal to 600 protons per second at the maximum rate, which is a reasonable value for a crista of this size [11], leads to a

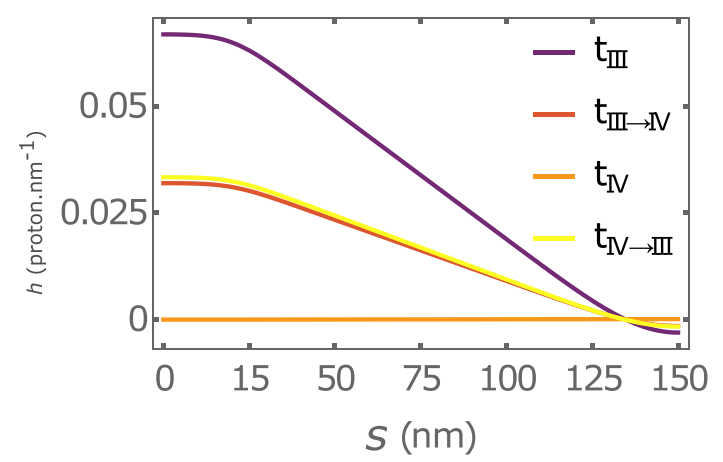

FIG. 3. Dynamics of the proton field along the cristae. The plots represent the field $h(s, t)$ solution of Eq. (3) for parameter values given in Table I and for $t_{\mathrm{IV}}=0[T], t_{\mathrm{IV} \rightarrow \mathrm{III}}=T / 4[T], t_{\mathrm{III}}=T / 2[T]$, $t_{\mathrm{III} \rightarrow \mathrm{IV}}=3 T / 4[T]$. The concentration $h(s, t)$ is expressed in proton per nm.

concentration of $8 \times 10^{-2}$ proton per nanometer. These plots are obtained in the case of an oscillating period much longer than the typical diffusion time along the tube, $2 \pi / \omega \gg L^{2} / D$.

\section{B. Model of the membrane}

To model the membrane of a mitochondrial crista, we start from the Helfrich model for elastic membranes. Developed by Wolfgang Helfrich in 1973 [21], this effective energy functional of a membrane takes into account molecular properties of lipid membranes such as the fluidity and the absence of in-plane shear stress but is written at a continuous coarsegrained scale. We modify the standard model by introducing $\mathrm{pH}$-dependent parameters,

$$
H=\int_{\Omega}\left\{\frac{1}{2} \kappa(s)\left[C-C_{0}(s)\right]^{2}+\sigma(s)\right\} d A,
$$

where $C$ is the local curvature of the surface and $d A$ the area of a surface element. The surface tension $\sigma(s)$, the bending modulus $\kappa(s)$, and the spontaneous curvature parameter $C_{0}(s)$ are assumed to depend linearly on the surface proton concentration as follows:

$$
\begin{gathered}
\kappa(s)=\kappa_{0}+h(s) \delta \kappa, \\
\sigma(s)=\sigma_{0}+h(s) \delta \sigma, \\
C_{0}(s)=C_{00}+h(s) \delta C_{0},
\end{gathered}
$$

with $h(s)$ defined in Eq. (1). For simplicity the Gaussian curvature is not considered here. In this chemico-mechanical model, the chemical reaction of ATP synthesis generates a dynamical field $h(s, t)$ on the membrane and will drive the deformation of the cylinder.

\section{DYNAMICAL EQUATIONS OF THE SURFACE DEFORMATION}

We consider an initial equilibrium state defined by a vanishing field $h(s, t)=0$ and a finite cylinder of length $L$ and of radius $R$. In this case, the Hamiltonian given in Eq. (A1) is restricted to the Helfrich model in which the spontaneous curvature $C_{00}$ is a phenomenological parameter, illustrating an 
asymmetry in the membrane. A nonvanishing, nonhomogeneous proton field $h(s)$ will lead to a deformed cylinder. The initial surface and the deformed surface, can be respectively parametrized by the three-dimensional vectors $\mathbf{X}_{0}$ and $\mathbf{X}_{t}$, defined as follows:

$$
\mathbf{X}_{t}=\mathbf{X}_{0}+\delta \mathbf{X}_{t}
$$

with

$$
\begin{aligned}
& \mathbf{X}_{0}=\left(\begin{array}{c}
R \cos (\theta) \\
R \sin (\theta) \\
z
\end{array}\right), \quad \delta \mathbf{X}_{t}=\left(\begin{array}{c}
u_{n}(z, t) \cos (\theta) \\
u_{n}(z, t) \sin (\theta) \\
u_{s}(z, t)
\end{array}\right) \\
& \text { with } \quad z \in[0, L], \quad \theta \in[0,2 \pi] .
\end{aligned}
$$

The surface is thus parametrized by the variables $z, \theta$ such that any point on this surface can be uniquely represented by a value of each of these parameters: $\mathbf{X}_{t}(s, \theta)$. The deformed state $\mathbf{X}_{t}$ is characterized by two fields $u_{n}(z, t)$ and $u_{s}(z, t)$ defined in Fig. 2(b). In this work, we consider only small deformations, i.e., $u_{s}(z, t) / R, u_{n}(z, t) / R$ much smaller than 1 . In the following, we will work in the intrinsic basis of the deformed surface represented in Fig. 2(b) and use the curvilinear abscissa $s$. To first order, the derivatives with respect to $z$ and $s$ of the deformation fields are equal: $\partial_{z} u_{i}(z, t)=\partial_{s} u_{i}(s, t)$, with $i=s, n$, and we will use the second ones.

\section{A. Some elements of differential geometry for an axisymmetric membrane}

We wish to describe the dynamics of the axisymmetric membrane $\mathbf{X}_{t}$ driven by the concentration field $h(t)$. Let us first introduce some terminology and results from differential geometry and their expressions to the first order in the deformation field. Note that to simplify the notations, we denote the fields $u_{n}(s, t)$ and $u_{s}(s, t)$ as $u_{n}$ and $u_{s}$ and the spatial derivative $\partial_{s} u_{i}(s, t)=u_{i}^{\prime}$, with $i=s, n$. The tangent vectors on the surface are defined as

$$
\begin{aligned}
& \mathbf{e}_{s}=\partial_{s} \mathbf{X}_{\mathbf{t}}=\left(\begin{array}{c}
u_{n}^{\prime} \cos (\theta) \\
u_{n}^{\prime} \sin (\theta) \\
1+u_{s}^{\prime}(s)
\end{array}\right), \\
& \mathbf{e}_{\theta}=\partial_{\theta} \mathbf{X}_{\mathbf{t}}=\left(\begin{array}{c}
\left.-\left(R+u_{n}\right) \sin (\theta) \$ R+u_{n}\right) \cos (\theta) \\
0
\end{array}\right) .
\end{aligned}
$$

The normal vector can be expressed as

$$
\mathbf{n}=\frac{e_{\theta} \wedge e_{s}}{\left|e_{\theta} \wedge e_{s}\right|}=\left(\begin{array}{c}
\cos (\theta) \\
\sin (\theta) \\
-u_{n}^{\prime}
\end{array}\right)
$$

and the metric of the surface is defined as $g_{a b}=\mathbf{e}_{\mathbf{a}} \cdot \mathbf{e}_{\mathbf{b}}$, with $(a=(\theta, s), b=(\theta, s))$ and is equal to

$$
g_{a b} \approx\left(\begin{array}{cc}
R^{2}+2 R u_{n} & 0 \\
0 & 1+2 u_{s}^{\prime}
\end{array}\right) .
$$

The curvature tensor, also known as the second fundamentalform, is defined as $K_{a b}=\mathbf{e}_{a} \cdot \partial_{b} \mathbf{n}$ using the convention that for a pointing outward normal vector, the curvature is positive [22]. It gives

$$
K_{a b}=\left(\begin{array}{cc}
R+u_{n} & 0 \\
0 & -u_{n}^{\prime \prime}
\end{array}\right)
$$

Finally, the sum $C=K_{s}^{s}+K_{\theta}^{\theta}$ of the principal curvatures can be written as

$$
C=\frac{1}{R}-\left(\frac{u_{n}}{R^{2}}+u_{n}^{\prime \prime}\right) .
$$

using $K_{b}^{a}=K_{a k} g^{k b}$, with $g^{a b}=\left(g_{a b}\right)^{-1}$.

Finally, we recall the expression of the covariant derivative of a tangential vector $x^{a} \mathbf{e}_{a}$,

$$
\nabla_{a} x^{b}=\partial_{a} x^{b}+\Gamma_{a c}^{b} x^{c}
$$

and of a tensor $t^{a b} \mathbf{e}_{a} \otimes \mathbf{e}_{b}$,

$$
\nabla_{a} t^{b c}=\partial_{a} t^{b c}+\Gamma_{a d}^{b} t^{d c}+\Gamma_{a d}^{c} t^{b d},
$$

where the Christoffel symbols can be written as

$$
\Gamma_{a b}^{s}=\left(\begin{array}{cc}
-R u_{n}^{\prime} & 0 \\
0 & u_{s}^{\prime \prime}
\end{array}\right), \quad \Gamma_{a b}^{\theta}=\left(\begin{array}{cc}
0 & \frac{u_{n}^{\prime}}{R} \\
\frac{u_{n}^{\prime}}{R} & 0
\end{array}\right) .
$$

To characterize the dynamical deformation of the tube, we introduce the flow velocity of the surface elements of the membrane,

$$
\mathbf{v}(s, t)=v_{s}(s, t) e_{s}+v_{n}(s, t) \mathbf{n},
$$

with $v_{s}=\partial_{t} u_{s}$ and $v_{n}=\partial_{t} u_{n}$. It is composed of an in-plane flow $\mathbf{v}_{s}=v_{s} \mathbf{e}_{s}$ and a term describing the deformation of the surface $\mathbf{v}_{n}=v_{n} \mathbf{n}$.

\section{B. Stress tensor acting on the deforming surface}

Next, we determine the stress tensor $\mathbf{f}$ acting on the surface $\mathbf{X}_{t}$. General surface stresses are complex objects that one can grasp by asking the question: "What forces should be exerted onto a membrane edge with unitary length to prevent it from shrinking?" [22]. In this case, the stress tensor is the sum of two contributions: a mechanical stress tensor $\mathbf{f}_{H}$ deriving from the Helfrich energy given in Eq. (A1) and a viscous stress tensor $\mathbf{f}_{\eta}$.

The mechanical stress tensor $\mathbf{f}_{H}$ can be written as a $3 \times 2$ tensor $[23,24]$ that can be decomposed into a surface stress tensor $f_{H}^{a b}$ generating forces tangent to the surface and a $2 \times 1$ tensor $\mathbf{f}_{H}^{n}=f_{H}^{a n} \mathbf{n} \times \mathbf{e}_{a}$ generating forces normal to the surface. To derive the expression of $\mathbf{f}$, we follow the approach developed by Guven and coworkers, presented in the Appendix. Instead of varying the shape of the membrane $\left(\mathbf{X}_{t} \rightarrow\right.$ $\left.\mathbf{X}_{t}+\delta \mathbf{X}_{t}\right)$ and explicitly tracking the changes of the intrinsic basis, the metric, the curvature, and the energy of the deformed membrane, this elegant approach enforces the geometric relations associated with the fundamental forms of the membrane by introducing Lagrange multipliers. We introduce 
the extended functional

$$
\begin{aligned}
H_{c}= & H+\int_{\Omega} \lambda^{a b}\left(g_{a b}-\mathbf{e}_{a} \cdot \mathbf{e}_{b}\right) d A \\
& +\int_{\Omega} \Lambda^{a b}\left(K_{a b}-\mathbf{e}_{a} \cdot \nabla_{b} \mathbf{n}\right) d A+\int_{\Omega} \mathbf{f}^{a} \cdot\left(\mathbf{e}_{a}-\nabla_{a} \mathbf{X}\right) d A \\
& +\int_{\Omega} \lambda_{\perp}^{a}\left(\mathbf{e}_{a} \cdot \mathbf{n}\right) d A+\int_{\Omega} \lambda_{n}\left(\mathbf{n}^{2}-1\right) d A
\end{aligned}
$$

in which $H$ is given in Eq. (A1), the matrices $\lambda^{a b}$ and $\Lambda^{a b}$ enforce the definition of metric and the curvature, $\mathbf{f}^{a}$ pins the basis vector to the tangent of the surface, $\lambda_{\perp}^{a}$ enforces the normal vector to be perpendicular, and $\lambda_{n}$ its normalization. The minimization of the Hamiltonian Eq. (21) with respect to the, now, 12 independent functions gives [25]

$$
\begin{gathered}
\mathbf{f}_{H}^{a b}=T^{a b}-\mathcal{H}^{a c} K_{c}^{b}, \\
f_{H}^{a n}=-\left(\nabla_{b} \mathcal{H}^{a b}\right),
\end{gathered}
$$

with

$$
\begin{gathered}
\mathcal{H}^{a b}=\frac{\delta \mathcal{H}}{\delta K_{a b}}, \\
T^{a b}=-\frac{2}{\sqrt{g}} \frac{\delta \sqrt{g} \mathcal{H}}{\delta g_{a b}},
\end{gathered}
$$

with $\mathcal{H}=\frac{1}{2} \kappa(s)\left[C-C_{0}(s)\right]^{2}+\sigma(s)$, the mechanical energy density of the membrane. (Details of the calculation are given in the Appendix).

In our case, the mechanical stress associated with the surface $\mathbf{X}_{t}$ depends both on the deformation fields $\left(u_{s}(s, t), u_{n}(s, t)\right)$ and on the concentration field $h(s, t)$. The surface stress tensor $f_{H}^{a b}$ and the normal stress tensor $f_{H}^{a n}$ expanded to first order in the fields can be expressed as

$$
\begin{aligned}
& f_{H}^{a b}=f_{H 0}^{a b}+f_{H 1}^{a b}, \\
& f_{H}^{a n}=f_{H 0}^{a n}+f_{H 1}^{a n},
\end{aligned}
$$

with

$$
f_{H 0}^{a b}=\left(\begin{array}{cc}
\frac{\kappa_{0}\left(1-X^{2}\right)-2 \sigma_{0} R^{2}}{2 R^{4}} & 0 \\
0 & -\frac{(1-X)^{2} \kappa_{0}}{2 R^{2}}-\sigma_{0}
\end{array}\right),
$$

and $f_{H 0}^{a n}=0$, and where the first-order part,

$$
\begin{aligned}
& f_{H 1}^{a b}=f_{h}^{a b}+f_{M}^{a b}, \\
& f_{H 1}^{a n}=f_{h}^{a n}+f_{M}^{a n},
\end{aligned}
$$

is the sum of a term $f_{1 h}^{a b}$ depending on the concentration field $h(s, t)$ and of a term $f_{1 M}^{a b}$ depending on the deformation fields $u_{n}(s, t), u_{s}(s, t)$.

Using the expression of the Hamiltonian given in Eq. (A1), the dependences of $\kappa, C_{00}$, and $\sigma$ on $h(s, t)$ given in Eqs. (7)(9) and the expression of the stress tensor given in Eqs. (29) and (A13), we derive the expression of the stress tensor to first order in the fields and find

$$
\begin{aligned}
& f_{1 M}^{a b}=\left(\begin{array}{cc}
\frac{\left[2 R^{2} \sigma_{0}+\left(2-X^{2}\right) \kappa_{0}\right] u_{n}(s)-R^{2} X \kappa_{0} u_{n}^{\prime \prime}(s)}{R^{5}} & 0 \\
0 & \frac{1-X}{R^{3}} \kappa_{0} u_{n}(s)+\frac{(1-X)^{2} \kappa_{0}+2 R^{2} \sigma_{0}}{R^{2}} u_{s}^{\prime}(s)
\end{array}\right), \\
& f_{1 M}^{a n}=\left(0, \frac{\kappa_{0}}{R^{2}} u_{n}^{\prime}(s)+\kappa_{0} u_{n}^{\prime \prime \prime}(s)\right), \\
& f_{1 h}^{a b}=\left(\begin{array}{cc}
-\frac{2 R^{2} \delta \sigma+2 R X \kappa_{0} \delta C_{0}-\left(1-X^{2}\right) \delta \kappa}{2 R^{5}} R h(s) & 0 \\
0 & \frac{2 R\left[(1-X) \kappa_{0} \delta C_{0}-R \delta \sigma\right]-(1-X)^{2} \delta \kappa}{2 R^{2}} h(s)
\end{array}\right), \\
& f_{1 h}^{a n}=\left(0, \frac{R \kappa_{0} \delta C_{0}-(1-X) \delta \kappa}{R} h^{\prime}(s)\right) .
\end{aligned}
$$

The stress tensor depending on the proton field involves both the field $h(s, t)$ in its tangential component $f_{1 h}^{a b}$ and its spatial derivative $h^{\prime}(s, t)$ in its normal component $f_{1 h}^{a n}$. The latter contribution will vanish for a constant field $h$. In fact, a constant field $h$ simply induces a renormalization of the parameters of the Helfrich model. By contrast, a spatially nonhomogeneous field $h(s, t)$ will generate forces on the normal direction that tend to pinch or expand the tube.

The viscous stress tensor for a deforming membrane is a surface tensor that can be obtained from the two-dimensional strain rate,

$$
v_{a b}=\frac{1}{2}\left(\nabla_{a} v_{b}+\nabla_{b} v_{a}\right)+K_{a b} v_{n}
$$

where $\mathbf{v}$ is given in Eq. (20), which is derived from the strain tensor of a three-dimensional fluid shell taken in the limit of a small thickness [26]. The viscous stress in compressible thin films can be expressed as

$$
f_{a b, \eta}=2 \eta_{s}\left(v_{a b}-\frac{1}{2} v_{c}^{c} g_{a b}\right)+\eta_{b} v_{c}^{c} g_{a b},
$$

which involves the two-dimensional strain rate $v_{a b}$ and phenomenological coefficients $\eta_{s}$ and $\eta_{b}$ that are the shear and bulk viscosity of the film. The viscous stress $\mathbf{f}_{\eta}=f_{a b, \eta} \mathbf{e}^{a} \times \mathbf{e}^{b}$ can be written as $2 \times 2$ matrix, whose components are given in covariant coordinates in Eq. (36) [22]. It yields the force generated by the flow within the membrane surface. Note that we do not account for the bulk viscosity of the fluid surrounding the membrane in our force balance. Indeed, its 
contribution can be neglected for strongly curved membrane buds [27] and tubes [28] with characteristic sizes smaller than a few micrometers, which is the appropriate regime for cristae.

In the absence of deformation, i.e., when the fields $v_{n}$, $v_{s}, u_{n}, u_{s}, h$ vanish, the viscous stress also vanishes and the equilibrium shape of the surface can be obtained by setting to zero the divergence of the stress tensor $f_{H, 0}^{a b}$ given in Eq. (28).

\section{Hydrodynamic equations for the tubular membrane}

We now consider the response of the membrane to a timevarying field, $h(s, t)$. In the absence of inertia, corresponding to the low-Reynolds number regime which is appropriate at the length scales considered, the dynamics of the system derives from the force balance, which can be written in the tangential basis of the surface as

$$
\begin{gathered}
\nabla_{a} f_{b}^{a}+K_{a b} f_{n}^{a}=0, \\
\nabla_{a} f^{a n}-K_{a b} f^{a b}=0,
\end{gathered}
$$

where the stress tensor $\mathbf{f}=\mathbf{f}_{H}+\mathbf{f}_{\eta}$ is the sum of the viscous and the mechanical stress tensor. The first equation, Eq. (37), corresponds to the force balance on the surface along $\mathbf{e}_{s}$ and $\mathbf{e}_{\theta}$. The second equation, Eq. (38), often called the shape equation of the surface, is the force balance in the normal direction. Expanding the covariant derivatives in Eqs. (37) and (38) and collecting the first-order terms in the fields $v_{n}, v_{s}, u_{n}$, $u_{s}, h$, we derive the hydrodynamic equations governing the time evolution of the system

$$
\begin{gathered}
\left(\eta_{s}+\eta_{b}\right) \partial_{s} v_{s}^{s}+\left(\eta_{b}-\eta_{s}\right) \partial_{s} v_{\theta}^{\theta}+\partial_{s} f_{H 1, s}^{s} \\
+\left(f_{H 0, s}^{s}-f_{H 0, \theta}^{\theta}\right) \Gamma_{s \theta}^{s}+R f^{n s}=0 \\
\left(\eta_{s}+\eta_{b}\right) \frac{v_{s}^{s}}{R}+\left(\eta_{b}-\eta_{s}\right) \frac{v_{\theta}^{\theta}}{R}+\partial_{s} f_{H 1}^{n s}-u_{s} f_{H 0}^{s s}-R f_{H 1}^{s s} \\
+u_{n}^{\prime \prime} f_{H 0}^{\theta \theta}=0 .
\end{gathered}
$$

The force balance along $\mathbf{e}_{\theta}$ vanishes for symmetry reasons. Replacing the mechanical tensors $f_{0 H}^{a b}, f_{1 H}^{a b}, f_{1 H}^{a n}$ by their expressions given in Eqs. (28), (29), and (A13), we finally obtain the dynamical equations of the system as a function of the velocity, displacement, and concentration fields:

$$
\begin{gathered}
a_{1} v_{n}^{\prime}+a_{2} v_{s}^{\prime \prime}+a_{3} h^{\prime}=0, \\
b_{1} v_{n}+b_{2} v_{s}^{\prime}+b_{3} u_{n}+b_{4} u_{n}^{\prime \prime}+b_{5} u_{n}^{\prime \prime \prime \prime}+b_{6} h+b_{7} h^{\prime \prime}=0,
\end{gathered}
$$

where the coefficients $a_{i},(i=1, \ldots, 7)$ and $b_{i}(i=1, \ldots, 3)$ are given in Appendix A2 and where $u_{i}^{\prime}$ and $v_{i}^{\prime}$ denote the spatial derivative $u_{i}^{\prime}=\partial_{s} u_{i}(s, t)$ and $v_{i}^{\prime}=\partial_{s} v_{i}(s, t)$ for $i=$ $s, n$.

\section{STATIC GREEN FUNCTION OF AN INFINITE MEMBRANE CYLINDER}

To gain physical insight into the model and the static solutions of Eqs. (39) and (40), we derive the static Green function of the system. We consider as a reference equilibrium state an infinite cylinder of radius $R$ and a vanishing field $h(s)=0$.

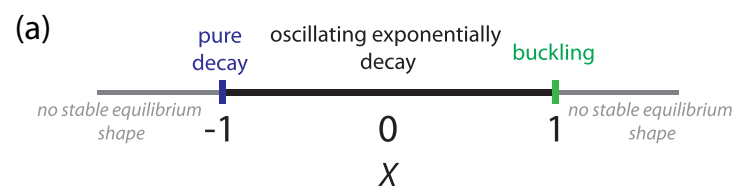

(b) 1.
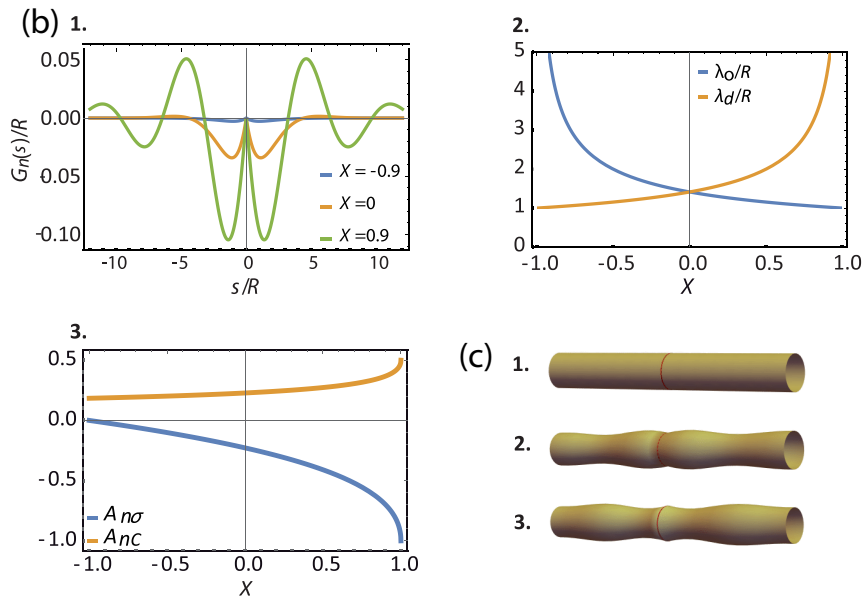

(c) 1.

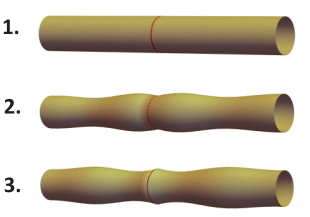

FIG. 4. (a) Stability diagram of a Helfrich cylinder as a function of $X=C_{00} R$. (b) $1 . G_{n}(s) / R$, Normalized Green functions for different values of $X=-0.9,0,0.9$ and for $\delta \sigma=0.15$ and $\delta C_{0}=0.2$. Characteristic lengths of oscillation $\lambda_{0}$ and decay $\lambda_{d}$ as a function of $X$. 3. Maximal amplitude of the deformations driven respectively by a variation of $\sigma, A_{n \sigma}$ and by a variation of $C_{0}, A_{n C}$. (c) Threedimensional representations of a cylinder (top) deformed by $h(s)=$ $\delta(s)$ for $\left(X=0.96, \delta \sigma=0.2, \delta \kappa=0, \delta C_{0}=0\right)$ (middle), and for $\left(X=0.96, \delta \sigma=-0.2, \delta \kappa=0, \delta C_{0}=0\right)$ (bottom). All quantities are dimensionless.

The shape equation for this system, given in Eq. (38), can be written as

$$
\sigma_{0}-\frac{\left(1-X^{2}\right) \kappa_{0}}{2 R^{2}}=0
$$

where we have employed the expression of $f_{H 0}^{a b}$ given in Eq. (28). Given that $R, \sigma$ and $\kappa$ are necessarily positive, this equation admits a solution for

$$
\left.X=C_{00} R \quad \in \quad\right]-1,1[.
$$

In this range [see Fig. 4(a)], the radius of the equilibrium cylinder $R$ can be expressed as a function of the parameters of the nonperturbed Helfrich model,

$$
R=\frac{1}{\sqrt{C_{00}^{2}+\frac{2 \sigma_{0}}{\kappa_{0}}}} .
$$

The stationary shape of a deformed cylinder associated with a perturbation field $h(s) \neq 0$ is given by the displacement fields $\left(u_{n}(s), u_{s}(s)\right)$, which are solutions of Eqs. (41) and (42) where $v_{n}$ and $v_{s}$ are set to zero. Replacing the coefficients $\left(a_{i}, b_{j}\right)$ by their expressions given in Appendix A 2, we obtain

$$
\begin{gathered}
{\left[\frac{4 R \sigma_{0} \delta C_{0}}{1+X}-2 \delta \sigma-\frac{\delta \kappa(1-X)^{2}}{R^{2}}\right] h^{\prime}(s)=0} \\
-\frac{2 \sigma_{0}}{R^{2}\left(1-X^{2}\right)}\left[u_{n}(s)+2 R^{2} X u_{n}^{\prime \prime}(s)+R^{4} u_{n}^{\prime \prime \prime \prime}(s)\right] \\
=\frac{2\left[(1+X) \delta \sigma-R \sigma_{0} \delta C_{0}\right]}{R\left(1-X^{2}\right)}\left[h(s)+R^{2} h^{\prime \prime}(s)\right] .
\end{gathered}
$$


The first equation corresponds to the force balance along the vector $\mathbf{e}_{s}$. The second equation is the shape equation. We observe that this system of equations does not depend on $u_{s}$. A nonhomogeneous perturbation field $h(s)$, which yields nonhomogeneous tension, bending rigidity, and spontaneous curvature, can lead to a stationary shape in the cylinder geometry only if the condition

$$
\left[\frac{4 R \sigma_{0} \delta C_{0}}{1+X}-2 \delta \sigma-\frac{\delta \kappa(1-X)^{2}}{R^{2}}\right]=0
$$

between the perturbation parameters, $\left(\delta \kappa, \delta \sigma, \delta C_{0}\right)$, is satisfied. Assuming that this condition holds, we express $\delta \kappa$ as a function of $\delta \sigma$ and $\delta X$,

$$
\delta \kappa=\frac{4 R \sigma_{0}}{(1-X)^{2}(1+X)} \delta C_{0}-\frac{2 R^{2}}{(1-X)^{2}} \delta \sigma .
$$

Let us now consider a localized perturbation in the proton concentration: $h(s)=h_{0} \delta(s)$ with $h_{0}=1$ introduced to dimension $h(s)$, and derive the Green function $G_{n}(s)$ yielding the deformation field $u_{n}(s)$ in response to this perturbation. Performing a Fourier transform of the shape equation given in Eq. (47) and introducing $\tilde{u}_{n}(q)=1 / 2 \pi \int d s e^{i q s} u_{n}(s)$, the normal deformation field in the Fourier space, we find

$$
\tilde{u}_{n}(q) \equiv \tilde{G}_{n}(q)=\frac{R\left(q^{2} R^{2}-1\right)\left[(1+X) \delta \sigma-R \sigma_{0} \delta C_{0}\right]}{\sigma_{0} \sqrt{2 \pi}\left(1-2 X q^{2} R^{2}+q^{4} R^{4}\right)} .
$$

Performing the inverse Fourier transform of $\tilde{G}_{n}$ given in Eq. (50), we obtain the expression of the Green function in real space,

$$
G_{n}(s)=G_{n} R \sin \left(\frac{|s|}{R} \sqrt{\frac{1+X}{2}}\right) \exp \left(-\frac{|s|}{R} \sqrt{\frac{1-X}{2}}\right)
$$

where we have introduced

$$
G_{n}=G_{n \sigma}(X) \frac{\delta \sigma}{\sigma}+G_{n C}(X) R \delta C_{0},
$$

with

$$
G_{n \sigma}(X)=-\frac{(1+X)}{\sqrt{2} \sqrt{1+X}}, \quad G_{n C}(X)=\frac{1}{\sqrt{2} \sqrt{1+X}}
$$

The deformation induced by a localized perturbation is thus an oscillating and exponentially decaying function, as shown in Fig. 4(b), panel 1. The characteristic lengths of oscillation $\lambda_{o}$ and decay $\lambda_{d}$ are given, respectively, by

$$
\begin{aligned}
& \lambda_{o}=R \sqrt{\frac{2}{1+X}}, \\
& \lambda_{d}=R \sqrt{\frac{2}{1-X}},
\end{aligned}
$$

for $X$ satisfying the condition given in Eq. (44).

Figure 4(b), panel 2, represents $\lambda_{o}$ and $\lambda_{d}$ as functions of $X$ for the range of possible equilibrium cylinders. The decay length $\lambda_{d}$ is an increasing function of $X$ that diverges for $X=1$. Thus, cylinders with larger spontaneous curvatures are deformed on a longer range by a heterogeneous $h$. The oscillation length $\lambda_{o}$, on the contrary, is a decreasing function of $X$.

The limit $X=1$ is associated with a phenomenon of buckling $\left(\lambda_{d}=0, \lambda_{o}=R\right)$, i.e., an infinite oscillating deformation wave. Indeed, $X=1$ corresponds to a cylinder with curvature along $e_{\phi}$ equal to the spontaneous curvature of the membrane. A sphere of radius $R$ is an equilibrium shape of such a membrane. A perturbation of the cylinder will tend toward such shapes by forming a succession of drops. Conversely, the limit $X=-1$ corresponds to a cylinder folded in the direction opposite to that of the spontaneous curvature. The deformation induced by a perturbation will then be purely decaying with a characteristic length $\lambda_{d}=R$, thus minimizing the energy of deformation.

The respective amplitudes $A_{n \sigma}, A_{n C}$ of the deformations induced by perturbations in $\sigma$ or in $C_{0}$, and defined as

$$
\begin{aligned}
& A_{n \sigma}(X)=G_{n \sigma}(X) \frac{G_{n}\left(s_{m}\right)}{G_{n}}, \\
& A_{n C}(X)=G_{n C}(X) \frac{G_{n}\left(s_{m}\right)}{G_{n}}
\end{aligned}
$$

are plotted with respect to $X$ in Fig. 4(b), panel 3. Here we have introduced $s_{m}=\sqrt{2} / \sqrt{1+X} \sin ^{-1}(\sqrt{X+1} / \sqrt{2})$, which is the coordinate of the maximum of $G_{n}(s) / G_{n}$. The absolute values of the amplitudes $A_{n \sigma}, A_{n C}$ are increasing functions of $X$ but possess opposite signs. An increase of the tension $[\delta \sigma>0, h(s)>0$ ] leads to a constriction of the cylinder (since $A_{n \sigma}<0$ ). On the other hand, an increase of the spontaneous curvature $\left(\delta C_{0}>0, h(s)>0\right)$ leads to a dilatation of the cylinder (since $A_{n C}>0$ ). A perturbation in the spontaneous curvature $\delta C_{0} R=0.1$ (respectively in the surface tension $\delta \sigma / \sigma=0.1$ ) will induce a variation of the radius of 5\% (respectively, 10\% ), for $X \rightarrow 1$. For $X$ negative or $0<X \ll 1$, the deformation induced by a variation of $h$ is negligible.

Figure 4(c) shows three-dimensional representations of the nonperturbed and of the perturbed cylinder for $X=0.9$, and with $\delta \sigma>0$ and $\delta \sigma<0$, respectively. This illustrates that the model proposed in Eq. (A1) can generate tubular membrane shapes of various curvature that can resemble mitochondrial cristae.

Using the expression of the Green function given in Eq. (51), we can find the stationary shapes generated by any proton concentration field $h(s)$ through

$$
u_{n}(s)=\int_{-\infty}^{\infty} h(x) G_{n}(s-x) d x
$$


(a)

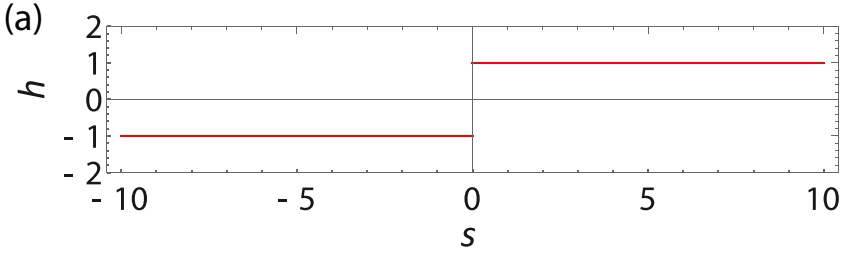

(b)

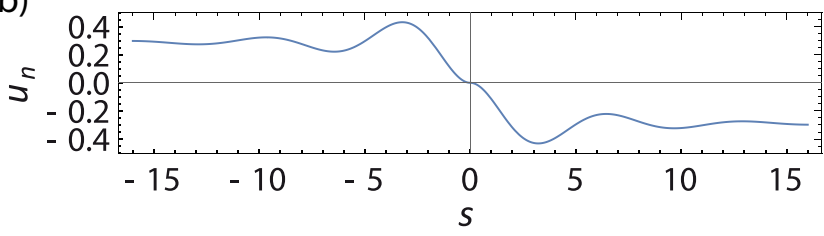

(c)

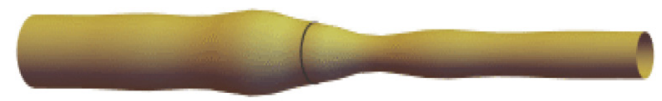

FIG. 5. Infinite cylinder submitted to a step of proton concentration. (a) Proton concentration $h(s)$ along the cylinder. (b) Deformation field $u_{n}(s)$ induced by a step of proton for $X=0.9$, $\delta \sigma=0.1, \delta C_{0}=-0.1$. (c) Three-dimensional representation of the corresponding shape. All quantities are dimensionless.

As a practical illustration, in Fig. 5, we consider a step function for $h(s)$,

$$
h(s)=-1 \quad \mathrm{~s}<0, \quad=1 \quad \mathrm{~s}>0
$$

[see Fig. 5(a)]. The corresponding deformation field $u_{n}(s)$ obtained using Eq. (57) is continuous, unlike the input step function, and is an odd function of $s$ that oscillates before reaching a plateau of constant value, as shown in Fig. 5(b). The resulting stationary shape of the tube, represented in Fig. 5(c), corresponds to two cylinders of different radii welded together through an oscillating neck.

\section{APPLICATION TO MITOCHONDRIA CRISTAE}

In this section, we describe the dynamical deformation of a finite tube of membrane of length $L$ submitted to the proton field $h(s, t)$ solution of Eq. (3) and represented in Fig. 3, which models a crista oscillating between state IV and state III.

To do so, we first specify the external mechanical forces exerted on the axisymmetric membrane. Here we do not take into account extra external pressures or viscous forces that could be applied to the membrane and we consider that a constant tension $f_{\text {ext }}$ is exerted by the rest of the mitochondria on the tubule boundary rings in $s=0$ and in $s=L$. This force,

$$
\begin{aligned}
& f_{\text {ext }}(0)=-\left[\sigma+\left(1-X^{2}\right) \frac{\kappa_{0}}{2 R^{2}}\right] \mathbf{e}_{s}, \\
& f_{\text {ext }}(L)=\left[\sigma+\left(1-X^{2}\right) \frac{\kappa_{0}}{2 R^{2}}\right] \mathbf{e}_{s}
\end{aligned}
$$

balances the effective tension of the undeformed Helfrich cylinder, defined by $\mathbf{X}_{0}$ in Eq. (11), and derives from the zero-order stress tensor $f_{H 0}$ given in Eq. (28). Consequently, the first-order forces deriving from the cylinder deformation vanish at the boundary of the tubule, and the first-order stress tensors satisfy

$$
\begin{aligned}
& f_{1, s}^{s}(0, t)=0, \quad f_{1, s}^{s}(L, t)=0, \\
& f_{H 1}^{s n}(0, t)=0, \quad f_{H 1}^{s n}(L, t)=0,
\end{aligned}
$$

where $f_{1, s}^{s}=f_{H 1, s}^{s}+f_{\eta, s}^{s}$, and where the expression of the stress is given in Eqs. (29), (A13), and (36). Moreover, the edges of the cylinder are assumed to be pinned in $s=0$ and $s=L$, which leads to a vanishing tangential velocity $v_{s}$ in $s=0$ and $s=L$,

$$
v_{s}(0, t)=0, \quad v_{s}(L, t)=0 .
$$

In order to facilitate the numerical resolution of the hydrodynamical equations given in Eqs.(41) and (42), the deformation fields $u_{n}(s, t), u_{s}(s, t)$ are expressed as function of the velocity fields $v_{n}(s, t)$ and $v_{s}(s, t)$ and of the deformation field at the time $t-d t$, using the backward Euler method and a discretization of the time with a time step $d t$,

$$
\begin{aligned}
& u_{s}^{t}=u_{s}^{t-d t}+d t \times v_{s}^{t}, \\
& u_{n}^{t}=u_{n}^{t-d t}+d t \times v_{n}^{t},
\end{aligned}
$$

where we have introduced the notation $\left(u_{i}^{t}, v_{i}^{t}\right)$ for $\left(u_{i}(s, t), u_{i}(s, t)\right)$. Inserting the expressions in Eqs. (63) and (64) the deformation fields into Eqs. (41) and (42) leads to the following coupled system of equations for $v_{s}^{t}$ and $v_{n}^{t}$ :

$$
\begin{gathered}
a_{1} \partial_{s} v_{n}^{t}+a_{2} \partial_{s}^{2} v_{s}^{t}+a_{3} \partial_{s} h^{t}=0, \\
\left(b_{1}+b_{3} \times d t\right) v_{n}^{t}+b_{4} \times d t \partial_{s} v_{n}^{t}+b_{5} \times d t \partial_{s}^{4} v_{n} \\
+b_{2} \partial_{s} v_{s}^{t}+b_{3} u_{n}^{t-d t}+b_{4} \partial_{s}^{2} u_{n}^{t-d t} \\
+b_{5} \partial_{s} u_{n}^{t-d t}+a_{6} h^{t}+a_{7} \partial_{s}^{2} h^{t}=0 .
\end{gathered}
$$

Assuming that the geometry of the system at the time $t-d t$ is known, the system to solve is a couple of ordinary differential equations in space for $v_{s}^{t}$ and $v_{n}^{t}$ for which the six necessary boundary conditions are given in Eqs. (60)-(62). The complete dynamic of the system is obtained by solving Eqs. (65) and (66) starting at $t=0$, when all the fields, $h^{0}, u_{i}^{0}, v_{i}^{0}$, vanish. Then, knowing the geometry of the system at the time $t-d t$, i.e., the deformation fields $u_{i}^{t-d t}$, and the concentration field at the time $t, h^{t}$, the velocities $\left(v_{s}^{t}(s), v_{n}^{t}(s)\right)$ are determined as solutions of Eqs. (65) and (66). Next, the deformation fields $u_{n}^{t}, u_{s}^{t}$ at time $t$ are calculated using Eqs. (63) and (64). The procedure can then be iterated to obtain the state of the system at time $t+d t$, and so forth. Further details on the numerical resolution are given in Appendix A 3 .

Figure 6(a) represents the shape of the tube for the oscillating proton field given in Fig. 3. The tube alternates between a quasinondeformed cylinder for a quasivanishing proton field (see Fig. 3) and a tubular invagination presenting a bump and a neck for the top proton flux. These oscillations are obtained in the regime of a fast mechanical relaxation time $\tau_{0}=2 \eta_{s} \eta_{b} / \sigma_{0}\left(\eta_{s}+\eta_{b}\right)$ compared to the oscillation period between state III and IV, $T=2 \pi / \omega$. It is thus the dynamics of the diffusive process that governs the shape change dynamics in this case. Figure 6(b) represents the deformation fields associated with these shapes. We compute the bump-to-neck 
(a)

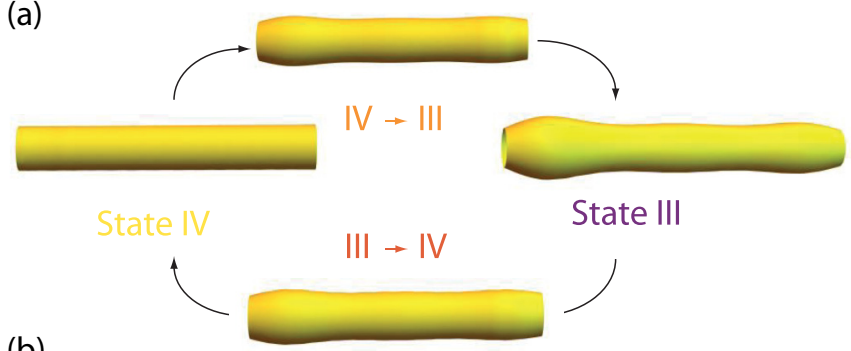

(b)
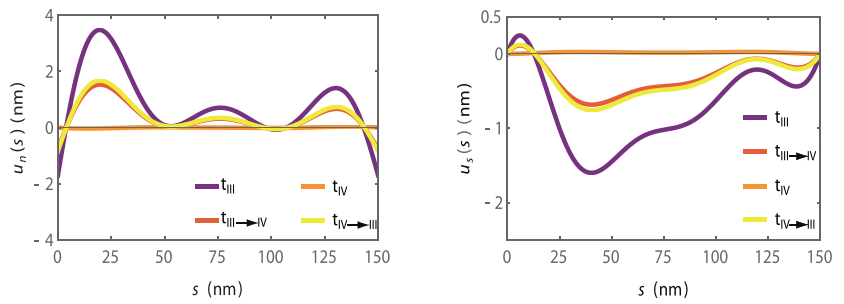

FIG. 6. Model crista oscillating between states IV and III. A finite membrane tube is submitted to the proton field $h(s, t)$ represented in Fig. 3. (a) Representation of the shape of the cylinder at different times of the period (the same ones as in Fig. 3). (b) Deformation fields $u_{n}(s, t)$ and $u_{s}(s, t)$ associated with these shapes (same colors as in Fig. 3). The plots are obtained for values given in Table I, $X=0.8, \delta \sigma=-0.35 \sigma$ per $h(s, t), \delta C_{0}=1.05 \operatorname{per} h(s, t)$.

ratio obtained in this framework and obtain 1.6. Measuring the projected sizes of the bumps and necks in state III on the eight best visible cristae on Fig. 1 yields an average bump-to-neck ratio of 1.9 (standard error of the mean: 0.3 ). The theoretical prediction is in agreement with the measures. This shows that the model developed in this work captures the salient features of the shape of the cristae in state III and IV observed experimentally and represented in Fig. 1 (regular tubes in state IV, bumpy tubes in state III).

\section{CONCLUSION}

Mitochondrial cristae are membrane protrusions that confine the ATP-synthesis machinery. Experimental observations have shown that the shape of these protrusions is coupled to the energetic state of mitochondria, as seen in Fig. 1. However, the underlying physical mechanisms controlling this coupling remain to be elucidated.

We considered the hypothesis that the shape of the invagination is driven by the flux of protons diffusing on the membrane. Indeed, the absence of cardiolipins in the crista membrane induces anomalous crista shapes, and local $\mathrm{pH}$ heterogenities induce crista-like deformations of GUVs comprising cardiolipins [17]. We described the mechanical properties of the mitochondrial crista membrane using a pH-dependent Helfrich model coupled to a diffusive proton concentration field on the surface. We first derived the stationary Green function of this system and showed that it can qualitatively reproduce the bumpy shapes of the mitochondrial invaginations observed experimentally. We then studied the dynamical shape change of the invagination for a membrane tubule subjected to an oscillating proton concentration field, modeling the oscillations between state IV (no ATP synthesis) and state III (high rate of ATP synthesis). We showed that, for appropriate values of the parameters, it can reproduce the salient features of the experimentally observed shapes of the mitochondrial cristae, oscillating between regular tubes in state IV and bumpy tubular protrusions in state III.

As a next step, for more realism, less symmetric morphologies closer to experimental observations of crista shapes, such as flat balloons with proton sinks on the rim and proton sources on flat zones, could be considered [20].

The phenomelogical model introduced here is a coarsegrained description of a membrane containing different lipids, proteins, etc. It will be interesting to consider a more microscopic model of the membrane to gain insight into the values of the parameters $\delta \kappa, \delta C_{0}, \delta \sigma$ of this model, and test whether realistic values yield membrane shapes resembling experimental observations.

Finally, a coupling between the shape and the concentration field could be introduced by considering the advection of the protons on the deformed membrane.

In vivo, the model could be tested with super-resolution imaging techniques by changing the energy demand and by monitoring crista morphology variation within mitochondria. Alternatively, experiments on biomimetic membranes can be performed where a local proton flux is introduced and membrane fluctuations are followed. ATP synthase and complexes of the respiratory chain can be co-reconstituted in liposomes to closely mimic the functioning of the respiratory chain [29]. Finally, proton diffusion along membranes mimicking the lipid composition of inner mitochondrial membranes can be estimated using lipid-anchored $\mathrm{pH}$ sensor fluorescent dye. Such experiments should bring measurements allowing us to test the ability of our model to predict crista morphology.

\section{APPENDIX}

\section{Covariant stress tensor for a membrane}

In this subsection we derive the stress tensor associated with the Hamiltonian

$$
H=\int_{\Omega}\left\{\frac{1}{2} \kappa(s)\left[C-C_{0}(s)\right]^{2}+\sigma(s)\right\} d A .
$$

We consider an infinitesimal deformation of the surface $\mathbf{X} \rightarrow \mathbf{X}+\delta \mathbf{X}$. Instead of tracking the variation of the intrinsic basis and the fundamental forms of the surface induce by this deformation, we enforce these geometrical constraints by introducing Lagrange multipliers, and we work with a generalized Hamiltonian $H_{c}$,

$$
\begin{aligned}
H_{c}=H & +\int_{\Omega} \lambda^{a b}\left(g_{a b}-\mathbf{e}_{a} \cdot \mathbf{e}_{b}\right) d A+\int_{\Omega} \Lambda^{a b}\left(K_{a b}-\mathbf{e}_{a} \cdot \nabla_{b} \mathbf{n}\right) \\
\times & d A+\int_{\Omega} \mathbf{f}^{a} \cdot\left(\mathbf{e}_{a}-\nabla_{a} \mathbf{X}\right) d A \\
& +\int_{\Omega} \lambda_{\perp}^{a}\left(\mathbf{e}_{a} \cdot \mathbf{n}\right) d A+\int_{\Omega} \lambda_{n}\left(\mathbf{n}^{2}-1\right) d A,
\end{aligned}
$$

in which the matrices $\lambda^{a b}$ and $\Lambda^{a b}$ enforce the definition of metric and the curvature, $\mathbf{f}^{a}$ pins the basis vector to the tangent of the surface, $\lambda_{\perp}^{a}$ enforces the normal vector to be perpendicular, and $\lambda_{n}$ is its normalization. The minimization of the 
Hamiltonian (A3) with respect to the, now, 12 independent functions gives the following equations:

$$
\begin{gathered}
\frac{\delta H_{c}}{\delta \mathbf{X}}=\nabla_{a} \mathbf{f}^{a}, \\
\frac{\delta H_{c}}{\delta \mathbf{e}_{a}}=-\mathbf{f}^{a}+\left(\Lambda^{a c} K_{c}^{b}+2 \lambda^{a b}\right) \mathbf{e}_{b}-\lambda_{\perp}^{a} \mathbf{n}, \\
\frac{\delta H_{c}}{\delta \mathbf{n}}=\left(\nabla_{b} \Lambda^{a b}+\lambda_{\perp}^{a}\right) \mathbf{e}_{a}+\left(2 \lambda_{n}-\Lambda^{a b} K_{a b}\right) \mathbf{n}, \\
\frac{\delta H_{c}}{\delta K_{a b}}=\Lambda_{a b}+\mathcal{H}^{a b} \\
\frac{\delta H_{c}}{\delta g_{a b}}=\lambda^{a b}+\lambda_{\rho} \rho \sqrt{g} \frac{g^{a b}}{2}+\lambda_{\phi} \rho \phi \sqrt{g} \frac{g^{a b}}{2}-\frac{1}{2} T^{a b},
\end{gathered}
$$

where $\mathcal{H}^{a b}$ and $T^{a b}$ are defined as the functional derivatives of the Hamiltonian density, $\mathcal{H}=\frac{1}{2} \kappa(s)\left[C-C_{0}(s)\right]^{2}+\sigma(s)$ with respect to the two fundamental forms:

$$
\begin{gathered}
\mathcal{H}^{a b}=\frac{\delta \mathcal{H}}{\delta K_{a b}}, \\
T^{a b}=-\frac{2}{\sqrt{g}} \frac{\delta \sqrt{g} \mathcal{H}}{\delta g_{a b}} .
\end{gathered}
$$

We notice that the Lagrange multiplier $\mathbf{f}^{a}$ obeys a conservation law $\left(\nabla_{a} \mathbf{f}^{a}=0\right)$. Using that Eqs. (A4)-(A8) vanish, we eliminate the Lagrange multipliers present in Eq. (A5) and find

$$
\mathbf{f}^{a}=\left(T^{a b}-\mathcal{H}^{a c} K_{c}^{b}\right) \mathbf{e}_{b}-\left(\nabla_{b} \mathcal{H}^{a b}\right) \mathbf{n} .
$$

The quantity $\mathbf{f}^{a}$ can be identified as the surface stress tensor [22,30]. The surface stress tensor is a $2 \times 3$ matrix and its coordinates in the intrinsic basis of the surface can be written as

$$
\begin{gathered}
\mathbf{f}_{H}^{a b}=T^{a b}-\mathcal{H}^{a c} K_{c}^{b}, \\
f_{H}^{a n}=-\left(\nabla_{b} \mathcal{H}^{a b}\right) .
\end{gathered}
$$

\section{Coefficients for dynamical force balance equations}

The full expression for the dynamical force balance equations Eqs. (41) and (42) can be obtained by inserting the coefficients, which are given as follows:

$$
\begin{aligned}
& a_{1}=\frac{\eta_{b}-\eta_{s}}{R}, \\
& a_{2}=\eta_{b}+\eta_{s},
\end{aligned}
$$

$$
\begin{aligned}
& a_{3}=-\frac{(1-X)^{2}}{2 R^{2}} \delta \kappa-\delta \sigma+\frac{2 R \sigma_{0}}{1+X} \delta C_{0}, \\
& b_{1}=\frac{\eta_{b}+\eta_{s}}{R^{2}} \\
& b_{2}=\frac{\eta_{b}-\eta_{s}}{R} \\
& b_{3}=\frac{2 \sigma_{0}}{R^{2}\left(1-X^{2}\right)} \\
& b_{4}=\frac{4 X \sigma_{0}}{1-X^{2}} \\
& b_{5}=\frac{2 R^{2} \sigma_{0}}{1-X^{2}} \\
& b_{6}=-\frac{\left(1-X^{2}\right)}{2 R^{3}} \delta \kappa+\frac{1}{R} \delta \sigma+\frac{2 X \sigma_{0}}{1-X^{2}} \delta C_{0}, \\
& b_{7}=\frac{1}{R\left(1-X^{2}\right)}\left[2 R^{3} \delta C_{0} \sigma_{0}-(1-X)^{2}(1+X) \delta \kappa\right] .
\end{aligned}
$$

Here $\kappa_{0}$ has already been substituted in terms of $\sigma_{0}, R$, and $X$ using the relation between the Helfrich parameters (45). If we set $a_{1}, a_{2}, b_{1}$, and $b_{2}$ to zero (i.e., no viscosities), we obtain the steady-state force balance equations for the Helfrich cylinder model.

\section{Details of the numerical resolution of hydrodynamics equations}

The system of equations (65) and (66) is solved numerically at time $t$ using the command NDSolve of the Mathematica. The solutions obtained for $v_{s}^{t}$ and $v_{n}^{t}$ are discretized in space on a regular grid with a step equal to $10^{-2}$ for a cylinder with radius $R=1$. The discrete spatial derivatives of the velocities $\left[v_{n}^{(i), \text { vect }},(i=1, \ldots, 4), v_{s}^{(j), \text { vect }},(j=1,2)\right]$ are derived on this grid.

The vectors are interpolated using polynomials of degree 18 to obtain analytical functions $\left(\partial_{s}^{i} v_{n}^{p o l}, \partial_{s}^{j} v_{s}^{p o l}, i=\right.$ $1, \ldots, 4, j=1, \ldots, 2)$, which are used to derive the deformation fields and their derivatives at the time step $t$,

$$
\begin{aligned}
& \partial_{s}^{i} u_{s}^{t}(s)=\partial_{s}^{i} u_{s}^{t-d t}+d t \times \partial_{s}^{i} v_{s}^{p o l}, \quad i=1, \ldots, 2, \\
& \partial_{s}^{j} u_{n}^{t}(s)=\partial_{s}^{j} u_{n}^{t-d t}+d t \times \partial_{s}^{j} v_{n}^{p o l}, \quad j=1, \ldots, 4 .
\end{aligned}
$$

[1] T. G. Frey and C. A. Manella, The internal structure of mitochondria, Trends Biochem. Sci. 25, 319 (2000).

[2] C. A. Mannella, Structure and dynamics of the mitochondrial inner membrane cristae, Biochim. Biophys. Acta (BBA)-Mol. Cell Res. 1763, 542 (2006).

[3] S. Koob and A. S. Reichert, Novel intracellular functions of apolipoproteins: The ApoO protein family as constituents of the Mitofilin/MINOS complex determines cristae morphology in mitochondria, Biol. Chem. 395, 285 (2014).
[4] D. M. Wolf, M. Segaxa, A. K. Kondadi, R. Anand, S. T. Bailey, A. S. Reichert, A. M. van der Bliek, D. B. Schackelford, M. Liesa, and O. S. Shirihai, Individual cristae within the same mitochondrion display different membrane potentials and are functionally independent, EMBO J 38, e101056 (2019).

[5] B. Chance and G. R. Williams, Respiratory enzymes in oxidative phosphorylation, III the steady state, JBC 217, 409 (1955).

[6] C. Wang, M. Taki, Y. Sato, Y. Tamura, H. Yaginuma, Y. Okada, and S. Yamaguchi, A photostable fluorescent marker for the superresolution live imaging of the dynamic structure of the 
mitochondrial cristae, Proc. Natl. Acad. Sci. USA 116, 15817 (2019).

[7] S. Cogliati, J. Enriquez, and L. Scorrano, Mitochondrial cristae: Where beauty meets functionality, Trends Biochem. Sci. 41, 261 (2016).

[8] P. Mitchell, Coupling of phosphorylation to electron and hydrogen transfer by a chemi-osmotic type of mechanism, Nature (London) 191, 144 (1961).

[9] R. B. Gennis, Proton dynamics at the membrane surface, Biophysical J. 110, 1909 (2016).

[10] A. M. Morelli, S. Ravera, D. Calzia, and I. Panfoli, An update of the chemiosmotic theory as suggested by possible proton currents inside the coupling membrane, Open Biol. 9, 180221 (2019).

[11] B. Rieger, W. Junge, and K. B. Busch, Lateral ph gradient between OXPHOS complex IV and $\mathrm{F}_{0} \mathrm{~F}_{1}$ ATP-synthase in folded mitochondrial membranes, Nat. Commun. 5, 3103 (2014).

[12] S. C. Al-Izzy, G. Rowlands, P. Sens, and M. S. Turner, HydroOsmotic Instabilities in Active Membrane Tubes, Phys. Rev. Lett. 120, 138102 (2018).

[13] H. Turlier, B. Audoly, J. Prost, and J.-F. Joanny, Furrow constriction in animal cell cytokinesis, Biophys. J. 106, 114 (2014).

[14] A. Mietke, F. Jülicher, and I. F. Sbalzarini, Self-organized shape dynamics of active surfaces, Proc. Natl. Acad. Sci. USA 116, 29 (2019).

[15] H. Berthoumieux, J.-L. Maître, C.-P. Heisenberg, E. K. Paluch, F. Jülicher, and G. Salbreux, Active elastic thin shell theory for cellular deformations, New J. Phys. 16, 065005 (2014).

[16] D. Acehan, A. Malhotra, Y. Xu, M. Ren, D. L. Stokes, and M. Schlame, Cardiolipin affects the supramolecular organization of ATP synthase in mitochondria, Biophys. J. 100, 2184 (2011).

[17] N. Khalifat, N. Puff, S. Bonneau, J.-B. Fournier, and M. I. Angelova, Membrane deformation under local $\mathrm{pH}$ gradient: Mimicking mitochondrial cristae dynamics, Biophys. J. 95, 4924 (2008).
[18] A.-F. Bitbol, L. Peliti, and J.-B. Fournier, Membrane stress tensor in the presence of lipid density and composition inhomogeneities, Eur. Phys. J. E 34, 53 (2011).

[19] A.-F. Bitbol, N. Puff, J.-B. Fournier Y. Sakuma, M. Imai, and M. I. Angelova, Lipid membrane deformation in response to a local pH modification: Theory and experiments, Soft Matter $\mathbf{8}$, 6073 (2012)

[20] K. M. Davies, M. Strauss, B. Daum, J. H. Heinz, D. Osiewacz, A. Rycovska, V. Zickermann, and W. Kühlbrandt, Macromolecular organisation of ATP synthase and complex I in whole mitochondria, Proc. Natl. Acad. Sci. USA 108, 14121 (2011).

[21] W. Helfrich, Elastic properties of lipid bilayers-Theory and possible experiments, Z. Naturforsch. C 28, 693 (1973).

[22] M. Deserno, Fluid lipid membranes: From differential geometry to curvature stresses, Chem. Phys. Lipids 185, 11 (2015).

[23] R. Capovilla and J. Guven, Stresses in lipid membranes, J. Phys. A 35, 6233 (2002).

[24] J.-B. Fournier, On the stress and torque tensors in fluid membranes, Soft Matter 3, 883 (2007).

[25] M. M. Müller, Theoretical studies of fluid membrane mechanics, Ph.D. thesis, Johannes Gutenberg-Universität Mainz (2007).

[26] A. Mietke, Dynamics of active surfaces, Ph.D. thesis, TU Dresden (2018).

[27] M. Arroyo and A. De Simone, Relaxation dynamics of fluid membranes, Phys. Rev. E 79, 031915 (2009).

[28] M. Rahimi and M. Arroyo, Shape dynamics, lipid hydrodynamics, and the complex viscoelasticity of bilayer membranes, Phys. Rev. E 86, 011932 (2012).

[29] J. Sjöholm, J. Bergstrand, T. Nilsson, R. Šachl, C. von Ballmoos, J. Widengren, and P. Brzezinski, The lateral distance between a proton pump and ATP synthase determines the ATPsynthesis rate, Sci. Rep. 7, 2926 (2017).

[30] J. Guven, Membrane geometry with auxiliary variables and quadratic constraints, J. Phys. A: Math. Gen. 37, L313 (2004). 